\title{
Comparison of radioguided excision with wire localization of occult breast lesions
}

\author{
A. Luini, S. Zurrida, G. Paganelli*, V. Galimberti, V. Sacchini, S. Monti, P. Veronesi, G. Viale $\dagger$ \\ and $\mathrm{U}$. Veronesi \\ Divisions of Senology, *Nuclear Medicine, $†$ Pathology, European Institute of Oncology, Milan, Italy \\ Correspondence to: Dr A. Luini, Senology Division, European Institute of Oncology, via G. Ripamonti 435, 20141 Milan, Italy
}

\begin{abstract}
Background: Clinically occult breast lesions are found with increasing frequency as a result of the widespread use of mammographic screening.

Methods: This study reports a new localization technique in which a small quantity of ${ }^{99 \mathrm{~m}} \mathrm{Tc}$-labelled colloidal albumin is inoculated directly into the lesion under stereotactic-radiographic or ultrasonographic guidance. Correct positioning of the inoculum is checked by scintigraphy. A $\gamma$ ray detection probe is then used to locate the lesion and guide its surgical removal. The results of this method in 30 patients were compared with those obtained using the established hook wire method in another 30 patients.

Results: In the wire localization group, the mean(s.d.) distance from the lesion centre to the specimen margin was $24 \cdot 5(0 \cdot 4)$ (range 15-45) $\mathrm{mm}$. For the radioguided group the figures were 13.8(0.3) (range 5-25) $\mathrm{mm}$. The mean(s.d.) lesion concentricity (difference between the maximum and minimum distance from the lesion border to specimen margin) was 6.2(0.4) (range 2-15) $\mathrm{mm}$ in the wire localization group and $3 \cdot 8(0 \cdot 2)$ (range 1-10) $\mathrm{mm}$ in the radioguided group.

Conclusion: Use of a $\gamma$ probe allowed rapid, easy and accurate removal of occult breast lesions. In comparison to the hook wire method, radioguided removal allows reduced excision volume and better lesion centring within the specimen.
\end{abstract}

Presented to the 20th Annual Breast Cancer Symposium in San Antonio, Texas, USA, 3-6 December 1997

Paper accepted 22 November 1998

British Journal of Surgery 1999, 86, 522-525

\section{Introduction}

Until recently non-invasive tumours formed a relatively small fraction (1-5 per cent) of diagnosed breast cancers ${ }^{1}$. The more widespread use of mammographic screening has increased the detection of preinvasive lesions. Recent publications indicate that $15-25$ per cent of breast cancers are intraductal carcinomas, most of which are clinically occult $^{2-9}$. Precise preoperative localization of an occult breast lesion is necessary before removal, yet none of the methods currently used can be regarded as perfect ${ }^{7,10-16}$. A new method for localizing non-palpable breast lesions, radioguided occult lesion localization (ROLL) has been developed at the European Institute of Oncology in Milan. The present study compared ROLL with the hook wire localization technique used previously.

\section{Materials and methods}

Between March 1996 and March 1998, 331 consecutive patients underwent excision biopsy using the ROLL procedure for suspicious non-palpable breast lesions detected mammographically. Data on the first 30 patients of this series were compared with those for the last 30 patients whose lesions were localized by means of the hook wire method.

\section{Radioguided occult lesion localization}

On the day before excision, patients were injected with particles of human serum albumin $10-150 \mu \mathrm{m}$ in diameter (Macrotec; Sorin Biomedica, Saluggia, Italy), labelled with approximately $3 \cdot 7 \mathrm{MBq}(0 \cdot 1 \mathrm{mCi}){ }^{99 \mathrm{~m}} \mathrm{Tc}$ at 
a specific activity of $74 \mathrm{MBq} / \mathrm{mg}$. The ${ }^{99 \mathrm{~m}} \mathrm{Tc}$ was always freshly eluted from a generator and, after labelling, the material was assayed for free technetium according to the supplier's instructions. Assays consistently showed more than 95 per cent of the radioactive metal bound to macroaggregate.

The labelled macroaggregate $(0.5 \mathrm{mg}$ in $0.2 \mathrm{ml}$ saline $)$ was inoculated, without local anaesthetic, into the centre of the suspicious lesion using stereotactic mammographic guidance when only microcalcifications were present, or ultrasonographic guidance for opacities. The technique was used only when the microcalcifications were closely grouped.

In all cases, front and lateral view planar scintigraphic images of the breast (exposure time $3 \mathrm{~min}$ ) were obtained with a $\gamma$ camera (GE-Star Camm 4000; GE Medical Systems, Milwaukee, Wisconsin, USA) soon after, and $5 \mathrm{~h}$ after administration. Correct localization of the tracer in the lesion, and at the inoculation point only, was checked by examining these images and superimposing them (appropriately enlarged) on the mammogram (Fig. 1). When ultrasonography was employed to image the lesion, correct tracer localization was verified as a change in the echogenicity of the lesion site.

Excision biopsy was performed next day guided by a hand-held $\gamma$ ray detection probe (C-Trak System; CareWise, Morgan Hill, California, USA) enclosed in a sterile glove. The $\gamma$ ray detection probe is an $18 \times 2-\mathrm{cm}$ stainless steel tube with an angled tip for enhanced manoeuvrability. The $\gamma$ rays detected by the probe are transduced into digital readout and acoustic signals. The intensity and frequency of the auditory signal are directly proportional to the level of radioactivity detected.

Using this probe the surgeon was able to find the skin projection of the lesion and decide the most appropriate incision. Having incised the skin, the area of maximum radioactivity, corresponding to the site of the lesion, was identified using the probe. The edges of the excision were defined as the locus of points surrounding the hot spot where radioactivity fell off sharply. After excising the specimen, the probe was used to check for residual radioactivity at the excision site; if this was present the excision was enlarged.
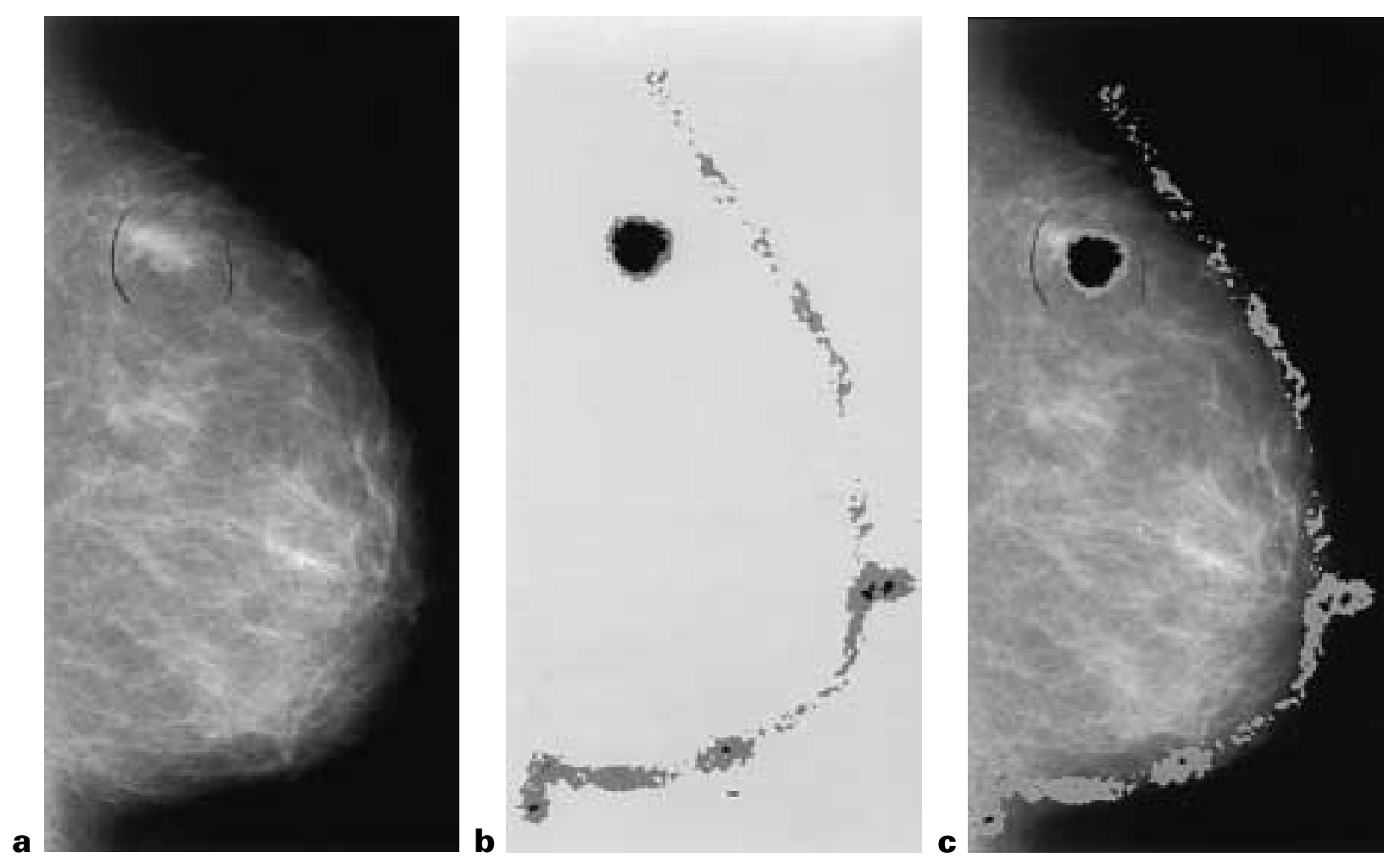

Fig. 1 a Mammogram showing a clinically occult lesion in the upper external quadrant of a right breast. b Lateral scintigraphic image of the injection position in the same patient. $\mathbf{c}$ The position of the hot spot exactly corresponds to the position of the lesion as revealed by mammography 


\section{Analysis of specimens}

Surgical specimens excised using the $\gamma$ ray detection probe and the guidewire were tagged with clips on one or more margins. To verify complete removal of the lesion, the specimen was placed in a special plexiglass container with the deep resection margin face down and radiographed. The concentricity of the lesion was calculated as the difference between the maximum and minimum distance from the border of the lesion to the margin of the specimen. The maximum distance from the lesion to the specimen margin was taken as an index of the quantity of tissue removed. Guidewire specimens were cut longitudinally along the wire and the distance from the margin to the needle apex was measured. The material was then sent for pathological examination.

\section{Results}

In the first 30 patients subjected to ROLL, postoperative scintigraphy of the removed specimen, as well as radiography, were used to verify the accuracy of removal. The mean distance from the centre of the lesion to the margin of the specimen was greater in the wire localization group than the ROLL group (Table 1). Lesions were also more concentrically placed within specimens in the ROLL group.

The pathological classification and subsequent treatments for the two groups are shown in Table 2. Lesions were classified according to the World Health Organization Histological Classification of Breast Tumours, as modified by Rosen and Oberman ${ }^{17}$.

\section{Subsequent experience}

A total of 331 patients have now had lesions excised using this procedure; 225 have been described previously ${ }^{18}$. Inoculation of labelled albumin caused at most minimal discomfort and no adverse reactions were observed. In 328 ( $99 \cdot 1$ per cent) of 331 cases the radioactive area coincided with the lesion which was located with the probe, both on the skin projection and within breast parenchyma. In the other three patients $(0.9$ per cent) preoperative scintigraphy revealed a mismatch (up to $2 \mathrm{~cm}$ ) between the radiological location of the lesion and the point of injection of the radioactivity. In both instances a wider excision surrounding the hot spot was performed; subsequent radiography of the specimen verified that the lesion had been removed completely.

\section{Dosimetry}

The mean(s.d.) absorbed dose to the inoculated area was estimated from measurements as 0.03(0.02) $\mathrm{mGy} / \mathrm{MBq}$.
Table 1 Results for the first 30 patients subjected to radioguided occult lesion localization and last 30 patients who underwent wire localization

\begin{tabular}{lll} 
& $\begin{array}{l}\text { Distance from lesion } \\
\text { centre to margin }(\mathrm{mm})\end{array}$ & $\begin{array}{l}\text { Lesion } \\
\text { concentricity }(\mathrm{mm})^{*}\end{array}$ \\
\hline ROLL $(\%)$ & $13 \cdot 8(0 \cdot 3)(5-25)$ & $3(0 \cdot 2)(1-10)$ \\
Guidewire localization $(\%)$ & $24 \cdot 5(0 \cdot 4)(15-45)$ & $6(0 \cdot 4)(2-15)$
\end{tabular}

Values are mean(s.d.) (range). *Difference between the maximum and minimum distance from the border of the lesion to the margin of the specimen. ROLL, radioguided occult lesion localization

Table 2 Comparison of treatments and histology in 30 patients subjected to radioguided occult lesion localization and 30 who underwent wire localization

\begin{tabular}{lcc} 
& ROLL (\%) & Wire (\%) \\
\hline Malignant & 59 & 55 \\
Ductal carcinoma in situ & 32 & 24 \\
Infiltrating cancer & 64 & 70 \\
Lobular carcinoma in situ & 5 & 5 \\
Benign & 41 & 45 \\
Quadrantectomy alone & 75 & 72 \\
Quadrantectomy with axillary & 25 & 28 \\
$\quad$ dissection & &
\end{tabular}

ROLL, radioguided occult lesion localization

Because of the small quantity of material injected and the fact that it was concentrated in tissue that was removed, the absorbed dose to the breast and other tissues was negligible.

External irradiation to staff was estimated from ionization chamber (Victoreen 450P; Victoreen, Cleveland, Ohio, USA) measurements of the exposure rate in air. The mean(s.d.) air kerma rates at 0,50 and $100 \mathrm{~cm}$ from the injection site immediately before surgery (around $20 \mathrm{~h}$ after injection) were $4 \cdot 5(1 \cdot 0), 1 \cdot 5(0 \cdot 4)$ and $0 \cdot 9(0 \cdot 3)$ Gy/h respectively. The mean(s.d.) absorbed dose to surgeon's hands was evaluated as $7 \cdot 5(5 \cdot 0) \mu \mathrm{Sv} / \mathrm{h}$. If 100 patients were treated per year, the absorbed dose to the surgeon's hands would correspond to $1 \cdot 5(1 \cdot 0)$ per cent and $0 \cdot 2(0 \cdot 1)$ per cent of the recommended limits for the general population $(50 \mathrm{mSv} / \mathrm{year})$ and exposed workers $\left(500 \mathrm{mSv} /\right.$ year) respectively ${ }^{19}$.

\section{Discussion}

Several techniques for the preoperative location of nonpalpable breast lesions have been developed. The choice of technique depends on lesion characteristics, particularly site, and the equipment available ${ }^{10-15}$. The most frequently used method is guidewire localization (Kopans wire, Homer wire or self-retaining anchor wire) under 
mammographic or ultrasonographic guidance to mark the lesion site ${ }^{15,16}$. This has the drawback that the needle introducing the wire may be deviated or become displaced. Needle introduction is traumatic and when the wire is wrongly positioned it cannot be removed to correct its position because of the risk of bleeding and because of the difficulty in achieving more accurate positioning. If the wire becomes displaced, as often occurs when the breast is very fatty, the excised specimen may not contain all of the lesion, forcing the surgeon to perform a wider resection. In very dense breasts guidewire placement is difficult.

Radioguided excision has proved reliable and effective in localizing occult lesions ${ }^{18}$. After excision, the probe is used for evaluation of the lesion centricity within the specimen. Previously postoperative mammograms were used to ensure that the entire lesion was removed. Using the present method the probe is used to check that the radioactivity is contained in the centre of the specimen and that none is left at the excision site. Subsequently, radiography is used to confirm that the lesion is completely contained within the specimen.

As with all localization techniques, care is required in the initial placement of the lesion marker; in this series the radioactive tracer was positioned correctly in $99 \cdot 1$ per cent of patients ${ }^{18}$. It was then a simple matter to locate the skin projection and perform the excision, in both cases guided by the probe.

Since the radioactivity is concentrated at a point, a very small dose is required for detection. Because of the small quantity of radioactivity the dose absorbed by the patient is small. For the same reason no more than routine precautions are required by the operating team. If 100 operations were performed per year the absorbed dose to the surgeon's hands would still be less than 2 per cent of the recommended limits for the general population ${ }^{19}$.

An advantage of the method is that the exact site of the radiolabelled lesion can be checked at any time during the operation using the probe. The surgeon can then determine the best incision to reach the lesion, independently of the site of inoculation. This results in high excision accuracy and centring of the lesion within the specimen, so that it is rarely necessary to enlarge the excision.

\section{Acknowledgements}

The authors thank E. Cassano, M. Cremonesi, F. Zerwes and G. Mazzarol for contributing to this study; and Don Ward for help with the English. This study was supported financially by the Italian Association for Cancer Research.

\section{References}

1 Page DL, Japaze H. Pathology of malignant lesions. Noninfiltrating (in situ) carcinoma. In: Bland KI, Copland EM III, eds. The Breast: Comprehensive Management of Benign and Malignant Diseases. Philadelphia, Pennsylvania: WB Saunders, 1991: 169-92.

2 Goedde TA, Frykberg ER, Crump JM, Lay SF, Turetsky DL, Linden SS. The impact of mammography on breast biopsy. Am Surg 1992; 58: 661-6.

3 Franceschi D, Crowe J, Zollinger R, Duchesneau R, Shenk R, Stefanek $\mathrm{G}$ et al. Breast biopsy for calcifications in nonpalpable breast lesions. A prospective study. Arch Surg 1990; 125: 170-3.

4 Griffen MM, Welling RE. Needle-localized biopsy of the breast. Surg Gynecol Obstet 1990; 170: 145-8.

5 Papatestas AE, Hermann D, Hermann G, Tsevdos C, Lesnick G. Surgery for nonpalpable breast lesions. Arch Surg 1990; 125: 399-402.

6 Rusnak CH, Pengelly DB, Hosie RT, Rusnak CN. Preoperative needle localization to detect early breast cancer. $A m \mathcal{F}$ Surg 1989; 157: 505-7.

7 Silverstein MJ, Gamagami P, Rosser RJ, Gierson ED, Colburn WJ, Handel $\mathrm{N}$ et al. Hooked-wire-directed breast biopsy and overpenetrated mammography. Cancer 1987; 59: 715-22.

8 Symmonds RE Jr, Roberts JW. Management of nonpalpable breast abnormalities. Ann Surg 1987; 205: 520-8.

9 Tubiana M, Holland R, Kopans DB, Kurtz JM, Petit JY, Rilke F et al. Commission of the European Communities 'Europe Against Cancer' Programme. European School of Oncology Advisory Report. Management of non-palpable and small lesions found in mass breast screening. Eur 7 Cancer 1994; 30A: $538-47$.

10 Berger SM, Curcio BM, Gershoncohen J, Isard HJ. Mammographic localization of unsuspected breast cancer. American Fournal of Roentgenology, Radium Therapy and Nuclear Medicine 1966; 96: 1046-52.

11 Hermann G, Janus G, Lesnick GJ. Percutaneous localisation of non palpable breast lesions. Breast 1983; 9: 4-6.

12 Frank HA, Hall FM, Steer ML. Preoperative localization of nonpalpable breast lesions demonstrated by mammography. N Engl 7 Med 1976; 295: 259-60.

13 Hall FM, Frank HA. Preoperative localization of nonpalpable breast lesions. AfR Am 7 Roentgenol 1979; 132: 101-5.

14 De Kopans DB, Luca S. A modified needle-hookwire technique to simplify preoperative localization of occult breast lesions. Radiology 1980; 134: 781.

15 Homer MJ. Nonpalpable lesion localization using a curvedend retractable wire. Radiology 1985; 157: 259-60.

16 Fornage BD, Faroux MJ, Simatos A. Breast masses: US-guided fine-needle aspiration biopsy. Radiology 1987; 162: 409-14.

17 Rosen PP, Oberman HA. Atlas of Tumour Pathology. Tumors of the Mammary Gland. 3rd series fascicle 7. Washington DC: Armed Forces Institute of Pathology, 1992.

18 Zurrida S, Galimberti V, Monti S, Luini A. Radioguided localization of occult breast lesions. The Breast 1998; 7: 11-13.

19 International Commission on Radiological Protection. Publication 60. Oxford: Pergamon Press, 1991. 